\title{
On the Impossibility of Group Membership*
}

\author{
Tushar Deepak Chandra ${ }^{\dagger} \quad$ Vassos Hadzilacos ${ }^{\ddagger} \quad$ Sam Toueg ${ }^{\S}$
}

\author{
Bernadette Charron-Bost $₫$
}

\begin{abstract}
We prove that the primary-partition group membership problem cannot be solved in asynchronous systems with crash failures, even if one allows the removal or killing of non-faulty processes that are erroneously suspected to have crashed.
\end{abstract}

\section{Introduction}

The problem of group membership has been the focus of much theoretical and experimental work on fault-tolerant distributed systems. A group membership protocol manages the formation and maintenance of a set of processes called a group. For example, a group may be a set of processes that are cooperating towards a common task (e.g., the primary and backup servers of a database), a set of processes that share a common interest (e.g., clients that subscribe to a particular newsgroup), or the set of all processes in the system that are currently deemed to be operational. In general, a process may leave a group because it failed, it voluntarily requested to leave, or it is forcibly expelled by other members of the group. Similarly, a process may join a group; for example, it may have been selected to replace a process that has recently left the group. A group membership protocol must manage such dynamic changes in some coherent way. In this paper we consider group membership protocols that ensure processes agree on the current membership of the group.

The group membership problem was first defined for synchronous systems by [Cri91]. Since then, the group membership problem for asynchronous systems has also been the

${ }^{*}$ Research partially supported by NSF grant CCR-9402894, DARPA/NASA Ames grant NAG-2-593, and a grant from the Natural Sciences and Engineering Research Council of Canada.

${ }^{\dagger}$ H2-L10, IBM T. J. Watson Research Center, 30 Saw Mill Road, Hawthorne, NY 10532, USA.

${ }^{\ddagger}$ Computer Systems Research Institute, University of Toronto, 6 King’s College Road, Toronto, Ontario, Canada M5S $1 \mathrm{~A} 1$.

$\S$ Department of Computer Science, Upson Hall, Cornell University, Ithaca, NY 14853, USA. This author worked on this paper while he was at INRIA (Project REFLECS) Rocquencourt, France.

`Laboratoire d'Informatique LIX, Ecole Polytechnique, 91128 Palaiseau Cedex, FRANCE. 
subject of intense investigation (e.g., [KT91, MPS91, RB91, VVR92, JFR93, vRBC ${ }^{+} 93$, BDGB94, BS94, DMS94, EMS95, MSMA94]). Yet, despite the wide interest that it has attracted and the numerous publications on this subject, the group membership problem for asynchronous systems is far from being understood: In particular, there is no agreed definition for this problem, and some of the most referenced formal definitions are unsatisfactory [ACBMT95].

Despite their differences, all versions of the group membership problem require some form of process agreement in systems with failures. Another well-known problem requiring agreement in spite of failures is Consensus. This problem, however, cannot be solved in asynchronous systems even if communication is reliable, only one process may fail, and it can do so only by crashing, i.e., if it stops executing steps [FLP85]. Since the purpose of group membership is to ensure some kind of agreement among processes (on the membership of a dynamically changing set), the potential for running into a similar impossibility result is obvious. On the other hand, group membership is different from Consensus in at least two ways:

- In group membership, a process that is suspected to have crashed can be removed from the group, or even killed, even if this suspicion is actually incorrect (e.g., the suspected process was only very slow). The [FLP85] model does not speak about process removals, and it does not directly model process killing (i.e., programcontrolled crashes).

- Consensus requires progress in all runs, while group membership allows runs that "do nothing" (for instance, "doing nothing" is desirable when no process wishes to join or leave the group, and no process crashes).

These differences appear to make group membership weaker than Consensus, and in fact the first one has been widely cited as a reason why group membership is solvable in asynchronous systems while Consensus is not [RB91, ADKM92, DKM93, DMS94, EMS95]. In this paper we prove that this is not so: We define a problem called WGM (for Weak Group Membership) that allows the removal of erroneously suspected processes from the group, and is subsumed by any reasonable definition of group membership, and show that WGM cannot be solved in asynchronous systems with failures. We first show the impossibility of WGM in systems where communication is reliable and where at most one process may crash, exactly as in [FLP85]. We then extend this negative result to systems that allow program-controlled process crashes.

One of the reasons why WGM is weak is that it makes no attempt to "track" failures: In contrast to most existing group membership services whose goal is to maintain a set of processes that are deemed to be operational (e.g., [RB91, JFR93, BDGB94, DMS94, MSMA94]), WGM does not link the membership of the group with actual or suspected crashes. Indeed, the only WGM requirement that ties the membership of the group to "reality" is a very weak and natural one: If a single process $p$ requests to leave the group, a WGM protocol should not preclude the possibility that $p$ is indeed the only process removed from the group. 
It is important to note that our result applies only to group membership services that attempt to maintain a single agreed view of the current membership of a group (e.g., [RB91, KT91, MPS91, MSMA94, HS95]). These are known as primary-partition group membership services and are intended for systems with no network partitions, or for systems that allow the group membership to change in at most one network partition, the "primary partition". So-called partitionable group membership services, which allow multiple views of the group to co-exist, have also been proposed [JFR93, vRBC ${ }^{+} 93$, BDGB94, DMS94, DMS95, EMS95]. Some remarks about such services are given at the end of this paper.

The rest of this paper is organized as follows. In Section 2, we define WGM and explain why it is weak. In Section 3, we show the impossibility of WGM in the [FLP85] model of asynchronous systems. The proof is patterned after the one given in [FLP85] with the exception of the starting lemma. In Section 4 we strengthen this impossibility result in various ways, and in Section 5 we extend it to systems that allow program-controlled crashes. Section 6 concludes the paper with some observations on the specification of group membership services.

\section{The WGM Problem}

We now describe WGM, a problem that captures a small fragment of what a realistic (primary-partition) group membership service should be able to provide. The impossibility of solving WGM in asynchronous systems with failures implies the impossibility of implementing more realistic group membership services in such systems. The informal specification of WGM given here is sufficient to understand the scope and meaning of our result; a formal specification in terms of the model of [FLP85] is given in the next section.

Consider a system with $n \geq 4$ processes $p_{1}, p_{2}, \ldots, p_{n}$, that can fail only by crashing, i.e., by stopping to execute steps. If a process never crashes, we say it is correct, otherwise it is faulty. We assume that initially all processes are in the same group $G$. More precisely, the first view of $G$ of every process is the set $V_{\text {init }}=\left\{p_{1}, p_{2}, \ldots, p_{n}\right\}$. At the beginning, some processes may wish to (voluntarily) leave group $G$. This, as well as actual or suspected crashes, may cause the membership of $G$ to change: For any $V \subseteq V_{\text {init }}$, we say that a process installs new view $V$ if it decides that $V$ is its second view of $G$ (such a decision is irrevocable). WGM satisfies the following properties:

1. If $p_{1}$, or $p_{2}$, or both (and no other process) wish to leave $G$, then at least one process eventually installs a new view, and no process installs a different new view.

2. For both $i \in\{1,2\}$, if $p_{i}$ is the only process that wishes to leave $G$, then it is possible that some process installs new view $V_{i}=V_{i n i t}-\left\{p_{i}\right\}$. In other words, there is at least one execution in which $p_{i}$ is the only process that wishes to leave $G$ and also the only one removed from $G$. 
Compared to a full-fledged group membership service, WGM is very weak. It imposes restrictions only on the first view change, and only if $p_{1}$ or $p_{2}$ initially wish to leave the group (it says nothing about subsequent view changes, or about what should happen if any process other than $p_{1}$ or $p_{2}$ wishes to leave $G$ ). Even in this case, only one process is required to install a new view. More importantly:

- WGM does not attempt to track actual (or even suspected) failures.

- The second requirement of WGM is natural for any reasonable group membership service. To see this, consider executions in which $p_{i}$ is the only process that wishes to leave $G$, and in which no failures or failure suspicions occur. It stands to reason that in at least one such execution, $p_{i}$ and only $p_{i}$ is removed from $G$.

- Except for the two executions mentioned in the previous item, there is no restriction on the size or content of the new view: In a single view change WGM is allowed to remove from group $G$ an arbitrary set of processes, including correct processes that did not wish to leave $G$. This is compatible with any group membership service that is allowed to remove from the group processes that are erroneously suspected to have crashed.

- There are no requirements on requests to join the group.

- There are no requirements on the broadcast or multicast of messages. In other words, there are no restrictions whatsoever on message delivery, and in particular on the way such deliveries interleave with view changes. ${ }^{1}$

\section{Impossibility of WGM in the [FLP85] Model}

We now formally define the WGM problem and show that it cannot be solved in the [FLP 85] model of distributed computing, namely, in asynchronous systems with reliable communication where at most one process may crash. The proof is very similar to the one of Fischer, Lynch and Paterson in [FLP85]. Essentially, the only difference stems from the fact that Consensus requires "termination" in all executions, while WGM does not. This affects principally one lemma - the only one whose proof we give in detail here.

The main features of the [FLP85] model are informally highlighted below.

- The system consists of $n$ processes $p_{1}, p_{2}, \ldots, p_{n}$, that communicate by sending messages over a completely connected point-to-point network. We assume $n \geq 4$.

- Processes execute steps at arbitrary speeds that can vary over time.

- At most one process may fail and can fail only by crashing.

\footnotetext{
${ }^{1}$ Most group membership services have such requirements. For example, the Virtual Synchrony property of ISIS requires the total ordering of multicasts with respect to view changes [BJ87].
} 
- Communication links are reliable but asynchronous: any message sent to a process that does not crash is received with a finite but arbitrary delay.

We expect readers to be familiar with the formalization of this model given in [FLP85]. We therefore do not repeat the definitions of terms already defined in that paper. Instead, we use boldface to indicate the first occurrence of a technical term borrowed from [FLP85]. When the definitions need to be adjusted to the new context (WGM instead of Consensus) we explain the necessary modifications.

Each process is modelled as an automaton. The internal state of each process $p$ contains, among other things, an input register $x_{p}$ and an output register $y_{p}$. The former holds a binary value indicating whether $p$ initially wishes to leave the group $G$ or not $\left(x_{p}=1\right.$ or $x_{p}=0$, respectively). (In [FLP85] $x_{p}$ indicates $p$ 's input value for Consensus.) The range of the output register $y_{p}$ is a subset of $V_{i n i t}=\left\{p_{1}, \ldots, p_{n}\right\}$. Initially, $y_{p}=V_{\text {init }}$, indicating that $p$ 's first view of $G$ contains every process. After its initial setting, $y_{p}$ may be written at most once. When (and if) $p$ writes $V \subseteq V_{\text {init }}$ into $y_{p}$, we say that $p$ installs new view $V$. (In [FLP85], the output register $y_{p}$ is initially "blank" and is written at most once with process $p$ 's decision value of the Consensus.)

An initial configuration can be uniquely identified by a bit vector of length $n$ that gives the value of $x_{p_{i}}$ for each $p_{i}, 1 \leq i \leq n$. Recall that WGM specifies the behavior of the system only when $p_{1}, p_{2}$ (or both) are the only processes that initially wish to leave $G$. Thus, the only initial configurations of interest are the ones corresponding to these three scenarios, namely, $C_{10}=\langle 100 \ldots 0\rangle, C_{01}=\langle 010 \ldots 0\rangle$, and $C_{11}=\langle 110 \ldots 0\rangle$.

The WGM problem in asynchronous systems is formally defined as follows.

1. For every $C \in\left\{C_{10}, C_{01}, C_{11}\right\}$, and every admissible run that starts from $C$, there is at least one process that installs a new view, and no process installs a different new view.

2. Starting from $C_{10}$ (respectively, $C_{01}$ ) there is at least one run such that some process installs new view $V_{1}=V_{\text {init }}-\left\{p_{1}\right\}$ (respectively, $V_{2}=V_{\text {init }}-\left\{p_{2}\right\}$ ).

Let $C$ be a configuration and let $\mathcal{V}_{C}$ be the set of views installed in configurations reachable from $C$. Formally, $\mathcal{V}_{C}=\left\{V \mid \exists C^{\prime}, \exists p: C^{\prime}\right.$ is reachable from $C$, and in $C^{\prime}$ $\left.y_{p}=V\right\}$. We say that $C$ is bivalent if $\left|\mathcal{V}_{C}\right| \geq 2$. (In [FLP85], $C$ is bivalent if the set of decision values of configurations reachable from $C$ contains two elements.)

The impossibility proof of [FLP85] is structured as follows. It first shows that a bivalent initial configuration exists. Then, starting from that bivalent configuration, it constructs an admissible run in which no process decides - contradicting the termination requirement of Consensus (which requires that every admissible run be a deciding run). This line of argument is not directly applicable here because in WGM not every admissible run is required to terminate: only those that start from $C_{10}, C_{01}$ or $C_{11}$ must result in the installation of a new view. Thus, it is not sufficient to show that some initial configuration is bivalent, but that one of $C_{10}, C_{01}$ or $C_{11}$ is. 
Lemma 1: $C_{10}, C_{01}$, or $C_{11}$ is bivalent.

Proof: If $C_{10}$ or $C_{01}$ is bivalent, we are done. Now suppose that neither one of $C_{10}$ or $C_{01}$ is bivalent. We show that in this case $C_{11}$ must be bivalent.

By the second property of WGM, there is a run that starts from $C_{10}$ such that a process installs $V_{1}$. Consider any admissible run $R$ that starts from $C_{10}$ in which $p_{2}$ takes no steps. By the first property of WGM, some process installs a new view $V$ in $R$. Since $C_{10}$ is not bivalent, $V=V_{1}$. Let $S$ be the schedule corresponding to $R$. Since $S$ contains no steps of $p_{2}$ and the only difference between $C_{10}$ and $C_{11}$ is the value of $p_{2}$ 's input register, $S$ is also applicable to $C_{11}$. Hence, from $C_{11}$ there is a reachable configuration in which some process installs new view $V_{1}$. By a symmetric argument, from $C_{11}$ there is a reachable configuration in which some process installs new view $V_{2}$. Therefore $C_{11}$ is bivalent.

Theorem 1: WGM is not solvable in asynchronous systems with crash failures, even if communication is reliable and at most one process may fail.

Proof (Sketch): Let $C$ be a bivalent configuration in $\left\{C_{01}, C_{10}, C_{11}\right\}$ (by Lemma 1 such a $C$ exists). We can now apply the techniques of [FLP85] to construct an infinite admissible run that starts from bivalent $C$ and remains bivalent forever: In this run, no process ever installs a new view - a contradiction to the first requirement of WGM.

\section{Strengthening the Impossibility Result}

Our specification of WGM allows processes to voluntarily request their removal from the group. This is a natural requirement which is compatible with the specification of the Strong Group Membership Problem (S-GMP) given in [RB91, Ric93, RB94]. In S-GMP a process $p$ may execute event faulty $_{p}(q)$ indicating that $p$ suspects that $q$ crashed. If this occurs, S-GMP requires that eventually either $p$ or $q$ are excluded from the group view. The possibility that $p=q$ is explicitly allowed, and hence $p$ executing fault $_{p}(p)$ amounts to a request for self-removal.

In any case, self-removal is not necessary to our impossibility result. To avoid selfremovals, we can simply: (1) reinterpret the meaning of the initial values, namely, $p_{1}$ has initial value 1 iff $p_{1}$ requests the removal of $p_{2}$ (say because $p_{1}$ suspects that $p_{2}$ has crashed), and $p_{2}$ has initial value 1 iff $p_{2}$ requests the removal of $p_{1} ;(2)$ redefine $V_{1}$ and $V_{2}$ to be $V_{\text {init }}-\left\{p_{2}\right\}$ and $V_{i n i t}-\left\{p_{1}\right\}$, respectively. The impossibility proof does not change.

The first property of WGM requires that no two processes, whether correct or not, disagree on the new view. This is akin to the Uniform Agreement property of [NT90]. One may wonder whether our impossibility result hinges on this uniformity requirement. It is easy to show that WGM remains unsolvable even if we only require that correct processes do not disagree.

The second property of WGM postulates the existence of two runs, one installing new view $V_{1}=V_{i n i t}-\left\{p_{1}\right\}$, and the other installing $V_{2}=V_{i n i t}-\left\{p_{2}\right\}$. This is a reasonable 
requirement but it can be weakened without affecting the impossibility result. An examination of the proof shows that all that is necessary is the existence of two runs (one starting from $C_{10}$ and one from $C_{01}$ ) that install distinct views, not necessarily $V_{1}$ and $V_{2}$.

\section{$5 \quad$ Impossibility of WGM Despite Process Killing}

Some group membership services have the ability to kill processes that are not faulty. For example, a process that is suspected of being faulty (say because it is very slow) can be removed from the group view and instructed to kill itself, even if this process is operational and did not actually fail. Alternatively, a process that has not succeeded in communicating with other processes for a long time, may decide to kill itself. It may appear that such "program-controlled" crashes of processes that hinder the progress of the algorithm make it easier for the remaining processes to install a new group view.

An example of program-controlled crashes appears in the $S$-GMP group membership protocol given in [RB91, Ric93]. In this protocol, if the process $p$ in charge of coordinating the update of group views comes to believe that a majority of the processes are faulty, it executes an event called crash. This causes $p$ to actually crash, i.e., to stop executing steps. This program-controlled crash is indistinguishable from a "genuine" crash that is due to, say, a hardware failure.

The model of [FLP85] does not quite capture such program-controlled crashes: in that model a process does not have the ability to stop taking steps if it wants to. Since we use the [FLP85] model to prove the impossibility of WGM (Theorem 1), one may ask whether our negative result still holds if program-controlled crashes are allowed.

To see why this question is pertinent, consider the proof of impossibility of WGM based on the [FLP85] model. This proof constructs an admissible run in which every process takes an infinite number of steps but never installs a new view. It is crucial for this construction that every process be capable of taking the next step at any point in time. This is true in the [FLP85] model, but is not true if we allow program-controlled crashes: once a process has executed a crash event, it cannot be required to take a next step. Thus, the argument of [FLP85] cannot be directly applied to systems that allow program-controlled crashes.

It turns out that the impossibility of WGM is not limited to the [FLP85] model: this negative result holds even if we allow program-controlled crashes. To show this, we must first model asynchronous distributed systems with program-controlled crashes. Let $\mathcal{M}$ denote the model of [FLP85]. Recall that in $\mathcal{M}$ each process is an automaton, and at most one process can stop executing steps at any state. Consider a model of computation denoted $\mathcal{M}_{c}$ that is identical to $\mathcal{M}$, except that the automaton associated with any process is now allowed to have a special state, called die, such that if a process enters that state, it stops executing steps. Thus, as in $\mathcal{M}$, model $\mathcal{M}_{c}$ allows at most one process to stop at any state (this models a genuine hardware crash), but unlike $\mathcal{M}$, in $\mathcal{M}_{c}$ any number of processes may stop at the die state (this models program-controlled crashes). Since $\mathcal{M}_{c}$ admits program-controlled crashes, the definition of admissible run is different from the 
one for $\mathcal{M}$ : In $\mathcal{M}$, an infinite run is admissible if at most one process stops executing steps, and every message sent to any process that takes an infinite number of steps is received. In $\mathcal{M}_{c}$, an infinite run is admissible if at most one process stops executing steps at a state different from die, and every message sent to any process that takes an infinite number of steps is received.

Theorem 2: WGM is not solvable in asynchronous systems with crash failures, even if communication is reliable and program-controlled crashes are allowed.

Proof: We show that WGM is not solvable in $\mathcal{M}_{c}$. The proof is based on Theorem 1 which shows that WGM is not solvable in $\mathcal{M}$.

Let $\mathcal{A}_{c}$ be any algorithm in model $\mathcal{M}_{c}$. $\mathcal{A}_{c}$ consists of a set of automata, one for each process in the system. Transform these automata into automata of $\mathcal{M}$ as follows: replace each die state with a nop state, such that if a process $p$ enters the nop state, $p$ continues to take steps and in every subsequent step that it takes, regardless of the message it receives (including the "null" message), $p$ remains in the nop state and does not send any messages. This transformation yields an algorithm $\mathcal{A}$ without die states, and so $\mathcal{A}$ is an algorithm in model $\mathcal{M}$.

We say that two runs are congruent if they start from the same initial configuration and each process installs a new view in one if and only if it installs the same view in the other. We claim that for each admissible run of $\mathcal{A}$ in $\mathcal{M}$ there is a congruent admissible run of $\mathcal{A}_{c}$ in $\mathcal{M}_{c}$, and vice-versa. From this claim and the specification of WGM, $\mathcal{A}_{c}$ solves WGM in $\mathcal{M}_{c}$ if and only if $\mathcal{A}$ solves WGM in $\mathcal{M}$. The theorem then follows immediately from Theorem 1. It now remains to show the claim.

Let $r$ be any admissible run of $\mathcal{A}$ in $\mathcal{M}$. Modify $r$ as follows: for each process $p$ that enters state nop, replace the step causing $p$ to enter nop for the first time into a step causing $p$ to enter the state die, and discard all subsequent nop steps of $p$. It is easy to verify that the resulting run is an admissible run of $\mathcal{A}_{c}$ in $\mathcal{M}_{c}$ that is congruent to $r$.

Conversely, let $r_{c}$ be any admissible run of $\mathcal{A}_{c}$ in $\mathcal{M}_{c}$. Let $D$ be the set of processes that enter state die in $r_{c}$. Let $r_{c}^{\prime}$ be the shortest prefix of $r_{c}$ such that all processes in $D$ have entered die, and $r_{c}^{\prime \prime}$ be the suffix of $r_{c}$ that follows $r_{c}^{\prime}$. Modify $r_{c}$ as follows: In the prefix $r_{c}^{\prime}$, for each process $p$ in $D$, replace the step causing $p$ to enter die into a step causing $p$ to enter state nop. In $r_{c}^{\prime \prime}$, after each step, insert a sequence of steps, one for each process in $D$; the message received in each of these steps is the oldest one sent to the process taking the step, or the "null" message if no such message exists. It is easy to verify that the resulting run is an admissible run of $\mathcal{A}$ in $\mathcal{M}$ that is congruent to $r_{c}$.

This completes the proof of the claim and hence of the theorem.

\section{Discussion}

A group membership service provides some kind of agreement among processes on the "current" membership of a group. This agreement requirement must be carefully specified so that two potentially conflicting goals are met: 
1. It must be weak enough to be solvable.

2. It must be strong enough to simplify the design of fault-tolerant distributed applications. In particular, it should not be satisfied by trivial or useless protocols.

For primary-partition group membership services, i.e., those that maintain agreement on a single view of the group at any one time, the impossibility of WGM indicates that these two goals are incompatible in asynchronous systems with failures, even if communication is reliable and processes may only crash. It is important to note that this impossibility result applies to group membership services that are allowed to remove from the group, and even kill, an arbitrary number of non-faulty processes that did not wish to be removed. Thus, contrary to a widespread view [RB91, ADKM92, DKM93, DMS94, EMS95], allowing the removal or killing of processes that are suspected to have crashed is not sufficient to make the primary-partition group membership problem solvable.

Note that the proof that WGM cannot be solved in asynchronous systems with failures hinges on the following liveness requirement of WGM: If $p_{1}$ or $p_{2}$ request to leave the group then a new view is eventually installed by at least one process in the system. Some implementations of primary-partition group membership do not satisfy this liveness requirement: They have runs that "block" forever, or remove or kill all processes. ${ }^{2}$ It is not clear, however, what liveness property (if any) such implementations do satisfy [ACBMT95].

In contrast to primary-partition group membership services, partitionable ones allow processes to disagree on the current membership of the group, i.e., several different views of the membership of the group may evolve concurrently and independently from each other [JFR93, vRBC ${ }^{+} 93, \mathrm{BDGB94}$, DMS94, DMS95, EMS95]. In particular, there may be several disjoint subsets of processes such that processes in each subset agree that they are the current members of the group. In other words, such group membership services allow group splitting (e.g., when the network partitions) and group merging (e.g., when communication between partitions is restored).

By allowing disagreement, such group membership services escape from the impossibility result of this paper. However, they run into another fundamental problem: their specification must be strong enough to rule out useless group membership protocols (in particular, protocols that can capriciously split groups into singleton sets) and yet it should be weak enough to remain solvable. To our knowledge, the design of such a specification is still an open problem [ACBMT95].

\section{Acknowledgements}

We are grateful to Lorenzo Alvisi and Prasad Jayanti for their insightful comments on an earlier version of this paper. We also thank Gerard LeLann and Pascale Minet for many helpful discussions on group membership services.

\footnotetext{
${ }^{2}$ For example, the implementation of $S$-GMP [RB91, Ric93] can crash all processes in the system before any new view is installed.
} 


\section{References}

[ACBMT95] Emmanuelle Anceaume, Bernadette Charron-Bost, Pascale Minet, and Sam Toueg. On the formal specification of group membership services. Technical Report 95-1534, Computer Science Department, Cornell University, Ithaca, New York 14853, August 1995.

[ADKM92] Yair Amir, Danny Dolev, Shlomo Kramer, and Dalia Malki. Membership algorithms for multicast communication groups. In Proceedings of the 6th International Workshop on Distributed Algorithms (WDAG - 6), (LCNS, 647), pages 292-312, November 1992.

[BDGB94] Özalp Babaoğlu, Renzo Davoli, Luigi-Alberto Giachini, and Mary Gray Baker. RELACS: a communications infrastructure for constructing reliable applications in large-scale distributed systems. BROADCAST Project deliverable report, 1994. Department of Computing Science, University of Newcastle upon Tyne, UK.

[BJ87] Kenneth P. Birman and Thomas A. Joseph. Exploiting virtual synchrony in distributed systems. In Proceedings of the 11th Annual Symposium Operating Systems Principles, pages 123-138, Austin, TX, November 1987.

[BS94] Özalp Babaoğlu and André Schiper. On group communication in large scale distributed systems. In Proceedings of the ACM SIGOPS European Workshop, Dagstuhl, Germany, September 1994.

[Cri91] Flaviu Cristian. Reaching agreement on processor group membership in synchronous distributed systems. Distributed Computing, 4(4):175-187, April 1991.

[DKM93] Danny Dolev, Shlomo Kramer, and Dalia Malki. Early delivery totally ordered multicast in asynchronous environment. In Proceedings of the 23th Annual International Symposium on Fault-Tolerant Computing, pages 544553, Toulouse, June 1993.

[DMS94] Danny Dolev, Dalia Malki, and Ray Strong. An asynchronous membership protocol that tolerates partitions. Technical Report CS94-6, Institute of Computer Science, The Hebrew University of Jerusalem, 1994.

[DMS95] Danny Dolev, Dalia Malki, and Ray Strong. A framework for partitionable membership service. Technical Report CS95-4, Institute of Computer Science, The Hebrew University of Jerusalem, 1995.

[EMS95] Paul D. Ezhilchelvan, Raimundo A. Macêdo, and Santosh K. Shrivastava. Newtop: a fault-tolerant group communication protocol. In Proceedings 
of the 15th International Conference on Distributed Computing Systems, Vancouver, BC, Canada, June 1995.

[FLP85] Michael J. Fischer, Nancy A. Lynch, and Michael S. Paterson. Impossibility of distributed consensus with one faulty process. Journal of the $A C M$, 32(2):374-382, April 1985.

[HS95] Matti A. Hiltunen and Richard D. Schlichting. Properties of membership services. In Proceedings of the 2nd International Symposium on Autonomous Decentralized Systems, Phoenix, AZ, April 1995.

[JFR93] Farnam Jahanian, Sameh Fakhouri, and Ragunathan Rajkumar. Processor group membership protocols: specification, design and implementation. In Proceeding of the 12th IEEE Symposium on Reliable Distributed Systems, pages 2-11, Princeton, October 1993.

[KT91] M. Frans Kaashoek and Andrew S. Tanenbaum. Group communication in the amoeba distributed operating system. In Proceedings of the 11th International Conference on Distributed Computer Systems, pages 222-230, Arlington, TX, May 1991.

[MPS91] Shivakant Mishra, Larry L. Peterson, and Richard D. Schlichting. A membership protocol based on partial order. In Proceedings of the IEEE International Working Conference on Dependable Computing For Critical Applications, pages 137-145, Tucson, AZ, February 1991.

[MSMA94] P.M. Melliar-Smith, Louise Moser, and Vivek Agrawala. Processor membership in asynchronous distributed systems. IEEE Transactions on Parallel and Distributed Systems, 5(5):459-473, May 1994.

[NT90] Gil Neiger and Sam Toueg. Automatically increasing the fault-tolerance of distributed algorithms. Journal of Algorithms, 11(3):374-419, September 1990.

[RB91] Aleta Ricciardi and Kenneth P. Birman. Using process groups to implement failure detection in asynchronous environments. In Proceedings of the 10th Annual ACM Symposium on Principles of Distributed Computing, pages 341-352, 1991. Also available as technical report 93-1328, Department of Computer Science, Cornell University.

[RB94] Aleta Ricciardi and Kenneth P. Birman. Process membership in asynchronous environments. Available by anonymous ftp from ftp.cs.cornell.edu in pub/aleta/AsyncMembService.ps, April 1994. 
[Ric93] Aleta Ricciardi. The group membership problem in asynchronous systems. PhD thesis, Department of Computer Science, Cornell University, USA, January 1993.

[vRBC $\left.{ }^{+} 93\right]$ Robbert van Renesse, Kenneth P. Birman, Robert Cooper, Bradford Glade, and Patrick Stephenson. The horus system. In Kenneth P. Birman and Robbert van Renesse, editors, Reliable Distributed Computing with the Isis Toolkit, pages 133-147. IEEE Computer Society Press, Los Alamitos, CA, 1993.

[VVR92] Paulo Verissimo, Werner Vogels, and Luis Rodrigues. Group orientation: a paradigm for modern distributed systems. In Proceedings of the $A C M$ SIGOPS 1992 Workshop, Mont Saint-Michel, France, 1992. 